PROCEEDINGS OF THE

AMERICAN MATHEMATICAL SOCIETY

Volume 137, Number 6, June 2009, Pages 1883-1890

S 0002-9939(09)09783-4

Article electronically published on January 15, 2009

\title{
REGULARITY AND SEGRE-VERONESE EMBEDDINGS
}

\author{
DAVID A. COX AND EVGENY MATEROV
}

(Communicated by Bernd Ulrich)

\begin{abstract}
This paper studies the regularity of certain coherent sheaves that arise naturally from Segre-Veronese embeddings of a product of projective spaces. We give an explicit formula for the regularity of these sheaves and show that their regularity is subadditive. We then apply our results to study the Tate resolutions of these sheaves.
\end{abstract}

\section{Regularity}

We will use the following generalized concept of regularity.

Definition 1.1. Fix a projective variety $X$ and line bundles $L, B$ on $X$ such that $B$ is generated by global sections. Then a coherent sheaf $\mathcal{F}$ on $X$ is $L$-regular with respect to $B$ provided that

$$
H^{i}\left(X, \mathcal{F} \otimes L \otimes B^{\otimes(-i)}\right)=0 \quad \text { for all } i>0 .
$$

Here $B^{\otimes(-i)}$ denotes the dual of $B^{\otimes i}$.

This notion of regularity is a special case the multigraded regularity for sheaves introduced in [6, which in turn is a modification of the regularity defined in 8 for multigraded modules over a polynomial ring.

We can relate Definition 1.1 to the Castelnuovo-Mumford regularity of a sheaf on projective space as follows. Suppose that $B$ is very ample and $\mathcal{L}=i_{*} \mathcal{F}$, where $i: X \rightarrow \mathbb{P}^{N}$ is the projective embedding given by $B$. Then, given an integer $p$, the isomorphism

$$
H^{i}\left(\mathbb{P}^{N}, \mathcal{L}(p-i)\right) \simeq H^{i}\left(X, \mathcal{F} \otimes B^{\otimes p} \otimes B^{\otimes(-i)}\right)
$$

shows that $\mathcal{F}$ is $B^{\otimes p}$-regular with respect to $B$ if and only if $\mathcal{L}$ is $p$-regular as a coherent sheaf on $\mathbb{P}^{N}$.

Now fix $r$-tuples $\underline{l}:=\left(l_{1}, \ldots, l_{r}\right)$ and $\underline{d}:=\left(d_{1}, \ldots, d_{r}\right)$ of positive integers. These give the product variety

$$
X:=\mathbb{P}^{l_{1}} \times \cdots \times \mathbb{P}^{l_{r}}
$$

of dimension $n:=\sum_{i=1}^{r} l_{i}$ and the $\underline{d}$-uple Segre-Veronese embedding

$$
\nu_{\underline{d}}: X \longrightarrow \mathbb{P}^{N}, \quad N=\prod_{k=1}^{r}\left(\begin{array}{c}
l_{k}+d_{k} \\
d_{k}
\end{array}\right)-1 .
$$

The $r$-tuples $\underline{l}$ and $\underline{d}$ will be fixed for the remainder of the paper.

Received by the editors May 29, 2008.

2000 Mathematics Subject Classification. Primary 13D02; Secondary 14F05, 16E05.

Key words and phrases. Regularity, Segre-Veronese embedding, Tate resolution.

(C)2009 American Mathematical Society 
Given $\underline{m}:=\left(m_{1}, \ldots, m_{r}\right)$, consider the line bundle

$$
\mathcal{O}_{X}(\underline{m})=\mathcal{O}_{X}\left(m_{1}, \ldots, m_{r}\right)
$$

defined by

$$
\mathcal{O}_{X}(\underline{m}):=p_{1}^{*} \mathcal{O}_{\mathbb{P}_{1}}\left(m_{1}\right) \otimes \cdots \otimes p_{r}^{*} \mathcal{O}_{\mathbb{P}^{l_{r}}}\left(m_{r}\right),
$$

where $p_{i}: \mathbb{P}^{l_{1}} \times \cdots \times \mathbb{P}^{l_{r}} \rightarrow \mathbb{P}^{l_{i}}$ is the projection. In this paper, we will study the regularity (in the sense of Definition 1.1) of $\mathcal{O}_{X}(\underline{m})$ with respect to the line bundles

$$
\begin{aligned}
L & :=\mathcal{O}_{X}(\underline{p})=\mathcal{O}_{X}\left(p_{1}, \ldots, p_{r}\right), p_{i} \in \mathbb{Z}, \\
B & :=\mathcal{O}_{X}(\underline{d})=\mathcal{O}_{X}\left(d_{1}, \ldots, d_{r}\right) .
\end{aligned}
$$

For any nonempty subset $J \subseteq\{1, \ldots, r\}$ let $l_{J}$ denote the sum $\sum_{j \in J} l_{j}$. Here is our first main result.

Theorem 1.2. Let $L=\mathcal{O}_{X}(\underline{p})$ and $B=\mathcal{O}_{X}(\underline{d})$ be as above. Then $\mathcal{O}_{X}(\underline{m})$ is $L$-regular with respect to $B$ if and only if

$$
\max _{k \in J}\left\{p_{k}+m_{k}+l_{k}-l_{J} d_{k}\right\} \geq 0
$$

for all nonempty subsets $J \subseteq\{1, \ldots, r\}$.

Proof. Observe that

$$
H^{i}\left(X, \mathcal{O}_{X}(\underline{m}) \otimes L \otimes B^{\otimes(-i)}\right)=H^{i}\left(X, \mathcal{O}_{X}(\underline{m}+\underline{p}-i \underline{d})\right)
$$

and also that

$$
H^{i}\left(X, \mathcal{O}_{X}(\underline{m}+\underline{p}-i \underline{d})\right)=\bigoplus_{i_{1}+\cdots+i_{r}=i} \bigotimes_{k=1}^{r} H^{i_{k}}\left(\mathbb{P}^{l_{k}}, \mathcal{O}_{\mathbb{P}^{l_{k}}}\left(m_{k}+p_{k}-i d_{k}\right)\right)
$$

by the Künneth formula. Since $H^{i_{k}}\left(\mathbb{P}^{l_{k}}, \mathcal{O}_{\mathbb{P}^{l} k}(j)\right)=0$ when $i_{k} \neq 0, l_{k}$, we may assume that $i=l_{J}$ for some $\emptyset \neq J \subseteq\{1, \ldots, r\}$ and that

$$
i_{k}= \begin{cases}l_{k}, & k \in J \\ 0, & k \notin J\end{cases}
$$

First suppose that (1.1) is satisfied for all $J \neq \emptyset$. Given such a $J$, pick $k \in J$ such that

$$
p_{k}+m_{k}+l_{k}-l_{J} d_{k} \geq 0 .
$$

Then $m_{k}+p_{k}-l_{J} d_{k} \geq-l_{k}$, so that $H^{l_{k}}\left(\mathbb{P}^{l_{k}}, \mathcal{O}_{\mathbb{P}^{l_{k}}}\left(m_{k}+p_{k}-l_{J} d_{k}\right)\right)=0$ by standard vanishing theorems for line bundles on projective space. By the above analysis, it follows easily that (1.2) vanishes for $i>0$.

Next suppose that

$$
\max _{k \in J}\left\{p_{k}+m_{k}+l_{k}-l_{J} d_{k}\right\}<0
$$

for some $J \neq \emptyset$. Among all such subsets $J$, pick one of maximum cardinality. For this $J$, we will prove that (1.2) is nonzero when $i=l_{J}$. By the Künneth formula, it suffices to show that

$$
\begin{array}{r}
H^{l_{k}}\left(\mathbb{P}^{l_{k}}, \mathcal{O}_{\mathbb{P}^{l_{k}}}\left(m_{k}+p_{k}-l_{J} d_{k}\right)\right) \neq 0, \quad k \in J, \\
H^{0}\left(\mathbb{P}^{l_{k}}, \mathcal{O}_{\mathbb{P} l_{k}}\left(m_{k}+p_{k}-l_{J} d_{k}\right)\right) \neq 0, \quad k \notin J .
\end{array}
$$

The first line of (1.4) is easy, since by Serre duality,

$$
H^{l_{k}}\left(\mathbb{P}^{l_{k}}, \mathcal{O}_{\mathbb{P}^{l_{k}}}\left(m_{k}+p_{k}-l_{J} d_{k}\right)\right) \simeq H^{0}\left(\mathbb{P}^{l_{k}}, \mathcal{O}_{\mathbb{P}^{l_{k}}}\left(-m_{k}-p_{k}+l_{J} d_{k}-l_{k}-1\right)\right)^{*} .
$$


This is nonzero provided $-m_{k}-p_{k}+l_{J} d_{k}-l_{k}-1 \geq 0$, which for $k \in J$ follows immediately from (1.3). For the second line of (1.4), suppose that $k \notin J$. By the maximality of $J$, we must have

$$
\max _{s \in J \cup\{k\}}\left\{p_{s}+m_{s}+l_{s}-l_{J \cup\{k\}} d_{s}\right\} \geq 0 .
$$

Note that $l_{J \cup\{k\}}=l_{J}+l_{k}$. If the maximum in (1.5) occurs at an element of $J$, say $s \in J$, then

$$
p_{s}+m_{s}+l_{s}-\left(l_{J}+l_{k}\right) d_{s} \geq 0,
$$

which is impossible since $s \in J$ and (1.3) imply that $p_{s}+m_{s}+l_{s}-I_{J} d_{s}<0$. Hence the maximum occurs at $k$, so that

$$
p_{k}+m_{k}+l_{k}-\left(l_{J}+l_{k}\right) d_{k} \geq 0 .
$$

This implies

$$
p_{k}+m_{k}-l_{J} d_{k} \geq l_{k} d_{k}-l_{k} \geq 0 .
$$

It follows that the second line of (1.4) is nonzero, as desired.

If we fix the sheaf $\mathcal{O}_{X}(\underline{m})$ and let $L=\mathcal{O}_{X}(\underline{p})$ vary over all $\underline{p} \in \mathbb{Z}^{r}$, we get the regularity set

$$
\operatorname{Reg}\left(\mathcal{O}_{X}(\underline{m})\right)=\left\{\underline{p} \in \mathbb{Z}^{r} \mid \mathcal{O}_{X}(\underline{m}) \text { is } \mathcal{O}_{X}(\underline{p}) \text {-regular for } B=\mathcal{O}_{X}(\underline{d})\right\} .
$$

This set is easy to describe. Given a permutation $\sigma$ in the symmetric group $S_{r}$, let $J(\sigma, k) \subseteq\{1, \ldots, r\}$ denote the subset

$$
J(\sigma, k)=\left\{\sigma(i) \mid i \geq \sigma^{-1}(k)\right\}, \quad 1 \leq k \leq r .
$$

Thus

$$
\begin{aligned}
J(\sigma, \sigma(1)) & =\{\sigma(1), \sigma(2), \ldots, \sigma(r)\} \\
J(\sigma, \sigma(2)) & =\{\sigma(2), \ldots, \sigma(r)\} \\
& \vdots \\
J(\sigma, \sigma(r)) & =\{\sigma(r)\} .
\end{aligned}
$$

Then define

$$
\underline{p}_{\sigma}=\left(-m_{1}-l_{1}+l_{J(\sigma, 1)} d_{1}, \ldots,-m_{r}-l_{r}+l_{J(\sigma, r)} d_{r}\right) .
$$

Proposition 1.3. The regularity set of $\mathcal{O}_{X}(\underline{m})$ is the union of $r$ ! translates of $\mathbb{N}^{r}$ given by

$$
\operatorname{Reg}\left(\mathcal{O}_{X}(\underline{m})\right)=\bigcup_{\sigma \in S_{r}}\left(\underline{p}_{\sigma}+\mathbb{N}^{r}\right) .
$$

Proof. First suppose that $\mathcal{O}_{X}(\underline{m})$ is $\mathcal{O}_{X}(\underline{p})$-regular for $B$. We build a permutation $\sigma \in S_{r}$ as follows. Theorem 1.2 tells us that (1.1) holds for all $J \neq \emptyset$. Hence, for $J=\{1, \ldots, r\}$, there is $k_{1}$ such that

$$
p_{k_{1}}+m_{k_{1}}+l_{k_{1}}-l_{J} d_{k_{1}} \geq 0 .
$$

Setting $\sigma(1)=k_{1}$, this becomes

$$
p_{k_{1}} \geq-m_{k_{1}}-l_{k_{1}}+l_{J\left(\sigma, k_{1}\right)} d_{k_{1}} .
$$

Applying (1.1) to $J=\{1, \ldots, r\} \backslash\{\sigma(1)\}$ gives $k_{2} \neq k_{1}$ with

$$
p_{k_{2}}+m_{k_{2}}+l_{k_{2}}-l_{J} d_{k_{2}} \geq 0 \text {. }
$$


Setting $\sigma(1)=k_{2}$, this becomes

$$
p_{k_{2}} \geq-m_{k_{2}}-l_{k_{2}}+l_{J\left(\sigma, k_{2}\right)} d_{k_{2}} .
$$

Continuing in this way gives $\sigma \in S_{r}$ with $\underline{p} \in \underline{p}_{\sigma}+\mathbb{N}^{r}$.

Conversely, let $\underline{p} \in \underline{p}_{\sigma}+\mathbb{N}^{r}$ and take any nonempty $J \subseteq\{1, \ldots, r\}$. Write $J=\left\{\sigma\left(i_{1}\right), \ldots, \sigma\left(\overline{i_{t}}\right)\right\}$ for $i_{1}<\cdots<i_{t}$. Then set $k=\sigma\left(i_{1}\right) \in J$ and observe that

$$
J \subseteq\left\{\sigma\left(i_{1}\right), \sigma\left(i_{1}+1\right), \ldots, \sigma(r)\right\}=J\left(\sigma, \sigma\left(i_{1}\right)\right)=J(\sigma, k) .
$$

This implies that $l_{J} \leq l_{J(\sigma, k)}$. Since $\underline{p} \in \underline{p}_{\sigma}+\mathbb{N}^{r}$, we obtain

$$
p_{k} \geq-m_{k}-l_{k}+l_{J(\sigma, k)} d_{k} \geq-m_{k}-l_{k}+l_{J} d_{k},
$$

so that $p_{k}+m_{k}+l_{k}-l_{J} d_{k} \geq 0$. This proves that (1.1) holds for $J$, and since $J \neq \emptyset$ was arbitrary, we have $\underline{p} \in \operatorname{Reg}\left(\mathcal{O}_{X}(\underline{m})\right)$ by Theorem 1.2 .

We next apply our results to study the Castelnuovo-Mumford regularity of $\mathcal{O}_{X}(\underline{m})$ under the projective embedding given by the Segre-Veronese map

$$
\nu_{\underline{d}}: \mathbb{P}^{l_{1}} \times \cdots \times \mathbb{P}^{l_{r}} \longrightarrow \mathbb{P}^{N}
$$

coming from $\mathcal{O}_{X}(\underline{d})$. This gives the sheaf $\mathcal{F}(\underline{m}):=\nu_{\underline{d} *} \mathcal{O}_{X}(\underline{m})$ on $\mathbb{P}^{N}$.

Theorem 1.4. The sheaf $\mathcal{F}(\underline{m})=\nu_{\underline{d} *} \mathcal{O}_{X}(\underline{m})$ is p-regular on $\mathbb{P}^{N}$ if and only if

$$
p \geq \max _{J \neq \emptyset}\left\{\min _{k \in J}\left\{l_{J}-\left\lfloor\frac{m_{k}+l_{k}}{d_{k}}\right\rfloor\right\}\right\} .
$$

Hence the regularity of $\mathcal{F}(\underline{m})$ is given by

$$
\operatorname{reg}(\mathcal{F}(\underline{m}))=\max _{J \neq \emptyset}\left\{\min _{k \in J}\left\{l_{J}-\left\lfloor\frac{m_{k}+l_{k}}{d_{k}}\right\rfloor\right\}\right\} .
$$

Proof. Let $B=\mathcal{O}_{X}(\underline{d})$. As noted earlier, $\mathcal{F}(\underline{m})$ is $p$-regular if and only if $\mathcal{O}_{X}(\underline{m})$ is $B^{\otimes p}$-regular with respect to $B$. Since $B^{\otimes p}=\mathcal{O}_{X}(p \underline{d})$, Theorem 1.2 implies that $\mathcal{F}(\underline{m})$ is $p$-regular if and only if

$$
\max _{k \in J}\left\{p d_{k}+m_{k}+l_{k}-l_{J} d_{k}\right\} \geq 0
$$

for all subsets $J \neq \emptyset$ of $\{1, \ldots, r\}$. This is equivalent to

$$
p \geq \min _{k \in J}\left\{l_{J}-\frac{m_{k}+l_{k}}{d_{k}}\right\}
$$

for all $J \neq \emptyset$. From here, the first assertion of the theorem follows easily, and the second assertion is immediate.

Example 1.5. When $r=2$, the usual Segre embedding $\nu: \mathbb{P}^{a} \times \mathbb{P}^{b} \rightarrow \mathbb{P}^{a b+a+b}$ gives $\mathcal{F}(k, l)=\nu_{*} \mathcal{O}_{\mathbb{P} a \times \mathbb{P}^{b}}(k, l)$. Since $\underline{d}=(1,1)$, Theorem 1.4 implies that

$$
\begin{aligned}
\operatorname{reg}(\mathcal{F}(k, l)) & =\max \{a-\lfloor k+a\rfloor, b-\lfloor l+b\rfloor, \min \{a+b-\lfloor k+a\rfloor, a+b-\lfloor l+b\rfloor\}\} \\
& =\max \{-k,-l, \min \{b-k, a-l\}\} \\
& =\max \{-\min \{k, l\}, \min \{b-k, a-l\}\} .
\end{aligned}
$$

This is the regularity formula from [4, Lem. 3.1]. 
Example 1.6. Let $\mathcal{I}$ be the ideal sheaf of $Y=\nu_{\underline{d}}(X) \subset \mathbb{P}^{N}$. Following [1], set $q_{k}=\left\lfloor\frac{l_{k}+1}{d_{k}}\right\rfloor$ and $q_{0}=\min _{1 \leq k \leq r}\left\{q_{k}\right\}$, and define

$$
\lambda= \begin{cases}n+2-q_{0}, & q_{0}=q_{k} \text { for some } k \text { with } d_{k} \mid l_{k}+1, \\ n+1-q_{0}, & \text { otherwise. }\end{cases}
$$

Recall that $n=\sum_{k=1}^{r} l_{k}=\operatorname{dim}(Y)$. Then [1, Lem. 3.4] asserts that $\mathcal{I}$ is $\lambda$-regular.

We can derive this from Theorem 1.4 as follows. We know that $\mathcal{I}$ is $\lambda$-regular if and only if $\mathcal{O}_{Y}$ is $(\lambda-1)$-regular. Note also that $\lambda$ can be defined more simply as

$$
\lambda=n+1-\min _{1 \leq k \leq r}\left\{\left\lfloor\frac{l_{k}}{d_{k}}\right\rfloor\right\} .
$$

If $J \neq \emptyset$, it follows easily that

$$
\lambda-1 \geq l_{J}-\min _{k \in J}\left\{\left\lfloor\frac{l_{k}}{d_{k}}\right\rfloor\right\} \geq l_{J}-\max _{k \in J}\left\{\left\lfloor\frac{l_{k}}{d_{k}}\right\rfloor\right\}=\min _{k \in J}\left\{l_{J}-\left\lfloor\frac{l_{k}}{d_{k}}\right\rfloor\right\} .
$$

By Theorem 1.4, we obtain $\lambda-1 \geq \operatorname{reg}(\mathcal{F}(\underline{0}))=\operatorname{reg}\left(\mathcal{O}_{Y}\right)$, so that $\lambda \geq \operatorname{reg}(\mathcal{I})$, as claimed.

It is also easy to see that $\lambda-1>\operatorname{reg}\left(\mathcal{O}_{Y}\right)$ can occur. For example, if $r=2$ and $\underline{d}=(1,1)$, then one can show without difficulty that

$$
\lambda-1=\max \left\{l_{1}, l_{2}\right\} \geq \min \left\{l_{1}, l_{2}\right\}=\operatorname{reg}\left(\mathcal{O}_{Y}\right) .
$$

\section{Subadditivity}

In this section we study how the regularity of $\mathcal{O}_{X}(\underline{m})$ and $\mathcal{O}_{X}\left(\underline{m}^{\prime}\right)$ compares to the regularity of the tensor product

$$
\mathcal{O}_{X}(\underline{m}) \otimes \mathcal{O}_{X}\left(\underline{m}^{\prime}\right)=\mathcal{O}_{X}\left(\underline{m}+\underline{m}^{\prime}\right) .
$$

We first consider regularity as defined in Definition 1.1 .

Theorem 2.1. Let $B=\mathcal{O}_{X}(\underline{d})$. If $\mathcal{O}_{X}(\underline{m})$ is $\mathcal{O}_{X}(\underline{p})$-regular for $B$ and $\mathcal{O}_{X}\left(\underline{m}^{\prime}\right)$ is $\mathcal{O}_{X}\left(\underline{p}^{\prime}\right)$-regular for $B$, then $\mathcal{O}_{X}\left(\underline{m}+\underline{m}^{\prime}\right)$ is $\mathcal{O}_{X}\left(\underline{p}+\underline{p}^{\prime}\right)$-regular for $B$.

Proof. We will use Theorem 1.2, Given a nonempty subset $J \subseteq\{1, \ldots, r\}$, it suffices to find $k \in J$ such that

$$
p_{k}+p_{k}^{\prime}+m_{k}+m_{k}^{\prime}+l_{k}-l_{J} d_{k} \geq 0
$$

Using (1.1) for this $J$ and the sheaves $\mathcal{O}_{X}(\underline{m})$ and $\mathcal{O}_{X}(\underline{p})$, we know that

$$
\max _{s \in J}\left\{p_{s}+m_{s}+l_{s}-l_{J} d_{s}\right\} \geq 0 .
$$

Hence we can find $k \in J$ such that

$$
p_{k}+m_{k}+l_{k}-l_{J} d_{k} \geq 0
$$

Then using (1.1) for $\{k\}$ and the sheaves $\mathcal{O}_{X}\left(\underline{m}^{\prime}\right)$ and $\mathcal{O}_{X}\left(\underline{p^{\prime}}\right)$, we also have

$$
p_{k}^{\prime}+m_{k}^{\prime}+l_{k}-l_{k} d_{k} \geq 0
$$

Since $l_{k} \leq l_{k} d_{k}$, this implies that

$$
p_{k}^{\prime}+m_{k}^{\prime} \geq 0
$$

Adding (2.2) and (2.3), we obtain the desired inequality (2.1).

Theorem 2.1 gives the following result concerning the Castelnuovo-Mumford regularity of the sheaves $\mathcal{F}(\underline{m})=\nu_{\underline{d} *} \mathcal{O}_{X}(\underline{m})$ on projective space. 
Theorem 2.2. Given any $\underline{m}, \underline{m}^{\prime} \in \mathbb{Z}^{r}$, we have

$$
\operatorname{reg}(\mathcal{F}(\underline{m}))+\operatorname{reg}\left(\mathcal{F}\left(\underline{m}^{\prime}\right)\right) \geq \operatorname{reg}\left(\mathcal{F}(\underline{m}) \otimes \mathcal{F}\left(\underline{m}^{\prime}\right)\right) .
$$

Proof. It suffices to show that if $\mathcal{F}(\underline{m})$ is $p$-regular and $\mathcal{F}\left(\underline{m}^{\prime}\right)$ is $p^{\prime}$-regular, then $\mathcal{F}\left(\underline{m}+\underline{m}^{\prime}\right)$ is $\left(p+p^{\prime}\right)$-regular. This follows immediately from Theorem 2.1 and the already-noted equivalence

$$
\mathcal{F}(\underline{n}) \text { is } q \text {-regular } \Longleftrightarrow \mathcal{O}_{X}(\underline{n}) \text { is } \mathcal{O}_{X}(q \underline{d}) \text {-regular for } B=\mathcal{O}_{X}(\underline{d}) \text {. }
$$

In general, regularity is not subadditive; i.e., given coherent sheaves $\mathcal{F}$ and $\mathcal{G}$ on $\mathbb{P}^{N}$, the inequality

$$
\operatorname{reg}(\mathcal{F})+\operatorname{reg}(\mathcal{G}) \geq \operatorname{reg}(\mathcal{F} \otimes \mathcal{G})
$$

may fail. Here is an example due to Chardin.

Example 2.3. Let $R=k[x, y, z, t]$ and consider the ideals $I_{n}=\left\langle z^{n}, t^{n}\right\rangle$ and $J_{m}=\left\langle x^{m-1} t-y^{m-1} z\right\rangle$. When $n, m \geq 3$, Example 1.13.6 of 2 implies that

$$
\operatorname{reg}\left(I_{n}\right)+\operatorname{reg}\left(J_{m}\right)=m+2 n-1<\operatorname{reg}\left(I_{n}+J_{m}\right)=m n-1 .
$$

As noted by Chardin [3], this remains true when we work in the larger ring $S=$ $k[x, y, z, t, u, v]$. The key point is that the ideals $I_{n}, J_{m}$, and $I_{n}+J_{m}$ are saturated in $S$.

Now let $\mathcal{F}$ and $\mathcal{G}$ be the coherent sheaves associated to $S / I_{n}$ and $S / J_{m}$ respectively. Then $\mathcal{F} \otimes \mathcal{G}$ is the sheaf associated to $S /\left(I_{n}+J_{m}\right)$. Since $I_{n}$ and $J_{m}$ are saturated in $S$, (2.5) easily implies that

$$
\operatorname{reg}(\mathcal{F})+\operatorname{reg}(\mathcal{G})=m+2 n-3<\operatorname{reg}(\mathcal{F} \otimes \mathcal{G})=m n-2
$$

when $n, m \geq 3$. This shows that subadditivity fails in general.

However, there are certain situations where (2.4) does hold, such as when $\mathcal{F}$ or $\mathcal{G}$ is locally free (see [7, Prop. 1.8.9]). Theorem 2.1 shows that the sheaves $\mathcal{F}(\underline{m})$ give another class of coherent sheaves for which regularity is subadditive.

\section{TATE RESOlutions}

By [5], a coherent sheaf $\mathcal{F}$ on the projective space $\mathbb{P}(W)$ has a Tate resolution

$$
\cdots \longrightarrow T^{p}(\mathcal{F}) \longrightarrow T^{p+1}(\mathcal{F}) \longrightarrow \cdots
$$

of free graded $E$-modules, $E=\bigwedge W^{*}$, with terms

$$
T^{p}(\mathcal{F})=\bigoplus_{i} \widehat{E}(i-p) \otimes H^{i}(\mathcal{F}(p-i)) .
$$

Here, $\widehat{E}=\operatorname{Hom}_{K}(E, K)$ is the dual over the base field $K$.

Standard vanishing theorems imply that

$$
T^{p}(\mathcal{F})=\widehat{E}(-p) \otimes H^{0}(\mathcal{F}(p)) \quad \text { for } p \gg 0 .
$$

Furthermore, if $\mathcal{F}=i_{*} \mathcal{E}$ for a locally free sheaf $\mathcal{E}$ on an irreducible Cohen-Macaulay subvariety $Y \hookrightarrow \mathbb{P}(W)$, then Serre duality and the same vanishing theorems imply that

$$
T^{p}(\mathcal{F})=\widehat{E}(n-p) \otimes H^{n}(\mathcal{F}(p-n)) \text { for } p \ll 0 .
$$

In this situation, we define

$$
\begin{aligned}
& p^{+}=\min \left\{p \mid T^{p}(\mathcal{F})=\widehat{E}(-p) \otimes H^{0}(\mathcal{F}(p))\right\} \\
& p^{-}=\max \left\{p \mid T^{p}(\mathcal{F})=\widehat{E}(n-p) \otimes H^{n}(\mathcal{F}(p-n))\right\} .
\end{aligned}
$$


In general, the differentials in the Tate resolution are hard to describe. The exceptions are the "horizontal" maps

$$
\widehat{E}(i-p) \otimes H^{i}(\mathcal{F}(p-i)) \longrightarrow \widehat{E}(i-p-1) \otimes H^{i}(\mathcal{F}(p+1-i)),
$$

which can be written down explicitly (see [5]). It follows that the Tate resolution is known for $p \geq p^{+}$and $p \leq p^{-}$. In other words, the interesting part of the Tate resolution lies in the range $p^{-} \leq p \leq p^{+}$.

For $X=\mathbb{P}^{l_{1}} \times \cdots \times \mathbb{P}^{l_{r}}$ and the Segre-Veronese embedding $\nu_{\underline{d}}: X \rightarrow \mathbb{P}^{N}=\mathbb{P}(W)$ coming from $\mathcal{O}_{X}(\underline{d})$, we have the sheaf $\mathcal{F}(\underline{m}), \underline{m}=\left(m_{1}, \ldots, m_{r}\right)$. When $r=2$ and $\underline{d}=(1,1)$, the Tate resolution, including differentials, is described in [4]. In the general case considered here, we restrict ourselves to giving explicit formulas for $p^{+}$ and $p^{-}$. This determines the length of the interesting part of the Tate resolution.

To state our formulas, recall that $\underline{l}=\left(l_{1}, \ldots, l_{r}\right)$ and $\underline{d}=\left(d_{1}, \ldots, d_{r}\right)$. We also set $\underline{1}=(1, \ldots, 1)$ and

$$
\underline{\widetilde{m}}=-\underline{m}+n \underline{d}-\underline{l}-\underline{1}=\left(-m_{1}+n d_{1}-l_{1}-1, \ldots,-m_{r}+n d_{r}-l_{r}-1\right) .
$$

Theorem 3.1. For the sheaf $\mathcal{F}(\underline{m})$, we have

$$
\begin{aligned}
& p^{+}=\operatorname{reg}(\mathcal{F}(\underline{m}))=\max _{J \neq \emptyset}\left\{\min _{k \in J}\left\{l_{J}-\left\lfloor\frac{m_{k}+l_{k}}{d_{k}}\right\rfloor\right\}\right\} \\
& p^{-}=-\operatorname{reg}(\mathcal{F}(\underline{\tilde{m}}))=-\max _{J \neq \emptyset}\left\{\min _{k \in J}\left\{\left\lceil\frac{m_{k}+1}{d_{k}}\right\rceil-l_{J^{c}}\right\}\right\},
\end{aligned}
$$

where $J^{c}$ denotes the complement of $J \subseteq\{1, \ldots, r\}$.

Proof. The equality $p^{+}=\operatorname{reg}(\mathcal{F}(\underline{m}))$ is immediate from (3.1) and the definition of regularity. Then Theorem [1.4 gives the desired formula for $p^{+}$. Since $\underline{\widetilde{m}}=$ $-\underline{m}+n \underline{d}-\underline{l}-\underline{1}$, Theorem 1.4 also implies that

$$
\begin{aligned}
\operatorname{reg}(\mathcal{F}(\underline{\tilde{m}})) & =\max _{J \neq \emptyset}\left\{\min _{k \in J}\left\{l_{J}-\left\lfloor\frac{\left(-m_{k}+n d_{k}-l_{k}-1\right)+l_{k}}{d_{k}}\right\rfloor\right\}\right\} \\
& =\max _{J \neq \emptyset}\left\{\min _{k \in J}\left\{\left\lceil\frac{m_{k}+1}{d_{k}}\right\rceil-l_{J^{c}}\right\}\right\},
\end{aligned}
$$

where the last equality follows easily using $l_{J}-n=-l_{J^{c}}$.

It remains to prove $p^{-}=-\operatorname{reg}(\mathcal{F}(\underline{\underline{\widetilde{m}}}))$. Observe that $p \leq p^{-}$if and only if

$$
H^{n-i}(\mathcal{F}(\underline{m})(p-(n-i)))=0 \text { for all } i>0 .
$$

By Serre duality on $X=\mathbb{P}^{l_{1}} \times \cdots \times \mathbb{P}^{l_{r}}$,

$$
\begin{aligned}
H^{n-i}(\mathcal{F}(\underline{m})(p-(n-i))) & =H^{n-i}\left(X, \mathcal{O}_{X}(\underline{m}+(p-(n-i) \underline{d}))\right. \\
& =H^{i}\left(X, \mathcal{O}_{X}(-\underline{m}-(p-n+i) \underline{d}-\underline{l}-\underline{1})\right)^{*} \\
& =H^{i}(\mathcal{F}(-\underline{m}+(n-p-i) \underline{d}-\underline{\underline{l}}-\underline{1}))^{*} \\
& =H^{i}(\mathcal{F}(\underline{\widetilde{m}})(-p-i))^{*},
\end{aligned}
$$

so that (3.2) is equivalent to

$$
H^{i}(\mathcal{F}(\underline{\underline{\widetilde{q}}})(-p-i))=0 \text { for all } i>0 .
$$

This vanishing is equivalent to $-p \geq \operatorname{reg}(\mathcal{F}(\underline{\widetilde{\widetilde{m}}}))$, so that

$$
p \leq p^{-} \Longleftrightarrow-p \geq \operatorname{reg}(\mathcal{F}(\underline{\tilde{m}})) \text {. }
$$

Hence $p^{-}=-\operatorname{reg}(\mathcal{F}(\underline{\underline{\widetilde{m}}}))$, as desired. 
Example 3.2. When $\underline{d}=(1, \ldots, 1)$ and $\underline{l}=(l, \ldots, l)$, we may assume that $\underline{m}=$ $\left(m_{1}, \ldots, m_{r}\right)$ with $m_{1} \leq \cdots \leq m_{r}$. Then one can show without difficulty that

$$
\begin{aligned}
& p^{+}=\max _{1 \leq i \leq r}\left\{(i-1) l-m_{i}\right\} \\
& p^{-}=\min _{1 \leq i \leq r}\left\{(i-1) l-m_{i}\right\}-1 .
\end{aligned}
$$

This makes it easy to compute $p^{+}-p^{-}$, which is the length of the interesting part of the Tate resolution. For instance, if $m_{1}=\cdots=m_{r-1}=0$ and $m_{r}=m$, where $m \geq(r-1) l$, then

$$
p^{+}-p^{-}=m-l+1
$$

which can be arbitrarily large. On the other hand, if $\underline{m}$ is more balanced, say $m_{1}=\cdots=m_{r}=m$, then

$$
p^{+}-p^{-}=(r-1) l+1,
$$

independent of $m$.

\section{ACKNOWLEDGEMENTS}

We are grateful to Jessica Sidman for suggesting that we recast the results of Sections 1 and 2 using the general notion of regularity given in Definition 1.1. Thanks also to Marc Chardin for his helpful comments about subadditivity and regularity. Finally, we are grateful to Nicolae Manolache for bringing the paper [1] to our attention.

\section{REFERENCES}

[1] Ş. Bărcănescu and N. Manolache, Betti numbers of Segre-Veronese singularities, Rev. Roumaine Math. Pures Appl. 26 (1981), 549-565. MR627814 (82j:13029)

[2] M. Chardin, Some results and questions on Castelnuovo-Mumford regularity, in Syzygies and Hilbert Functions (I. Peeva, ed.), Lecture Notes in Pure and Appl. Math 254, Chapman \& Hall/CRC, Boca Raton, FL, 2007, 1-40. MR2309925 (2008c:13023)

[3] M. Chardin, private communication.

[4] D. Cox and E. Materov, Tate Resolutions for Segre Embeddings, preprint, arXiv:0711.0550.

[5] D. Eisenbud, G. Fløystad and F.-O. Schreyer, Sheaf cohomology and free resolutions over exterior algebras, Trans. Amer. Math. Soc. 355 (2003), 4397-4426. MR.1990756 (2004f:14031)

[6] M. Hering, H. Schenck and G. Smith, Syzygies, multigraded regularity and toric varieties, Compos. Math. 142 (2006), 1499-1506. MR2278757 (2007k:13025)

[7] R. Lazarsfeld, Positivity in Algebraic Geometry. I, Ergebnisse der Mathematik 48, Springer, 2004. MR2095471 (2005k:14001a)

[8] D. Maclagan and G. Smith, Multigraded Castelnuovo-Mumford regularity, J. Reine Angew. Math. 571 (2004), 179-212. MR2070149 (2005g:13027)

Department of Mathematics and Computer Science, Amherst College, Amherst, MasSACHUSETTS 01002-5000

E-mail address: dac@cs.amherst.edu

Department of Mathematics and Statistics, University of Massachusetts, Amherst, MASSACHUSETTS 01003-9305

Current address: Fachbereich Mathematik und Informatik, Institut für Mathematik, Freie Universität Berlin, Arnimallee 3, D-14195 Berlin, Germany 